\title{
A scoring system is an effective tool for predicting central lymph node metastasis in papillary thyroid microcarcinoma: a case-control study
}

Ye-feng Cai ${ }^{1}$, Qing-xuan Wang ${ }^{1}$, Chun-jue Ni ${ }^{2}$, Xiang-jian Zhang ${ }^{1}$, En-dong Chen ${ }^{1}$, Si-yang Dong ${ }^{1}$, Hua-min Zheng ${ }^{3}$, Xiao-hua Zhang ${ }^{1}$ and Quan $\mathrm{Li}^{\mathrm{i}^{*}}$

\begin{abstract}
Background: The purpose of this study was to evaluate the clinicopathologic and ultrasonographic (US) characteristics and establish an effective scoring system for predicting central lymph node metastasis (CLNM) in papillary thyroid microcarcinoma (PTMC).

Methods: A total of 498 patients with PTMC who underwent total thyroidectomy or lobectomy with therapeutic central lymph node dissection (CLND) were enrolled. Univariate and multivariate analyses were performed to find the independent predictors for CLNM based on clinicopathological and US characteristics. Using the standardized regression coefficient, a 10-point score system was constructed in line with these independent predictors. Then, the scoring system was evaluated for the diagnostic value in predicting CLNM.

Results: Tumor location (the lower polo), tumor size (>5 mm), extrathyroidal extension, margin (no well-defined), display of enlarged lymph node, and contact of $>25 \%$ with the adjacent capsule were independent predictors for CLNM. Verifying the scoring system, a cutoff value of 5 points was found to be the best prediction for CLNM, the sensitivity and specificity were 64.7 and $80.5 \%$, respectively, and the positive and negative predictive values were 77.3 and $69.0 \%$, respectively.

Conclusions: The points $\leq 5$ could be considered as a low risk for CLNM, and the points $>5$ could be identified as a high risk for CLNM. More advanced diagnostic approaches and prophylactic CLND are needed for patients with the points $>5$.
\end{abstract}

Keywords: Thyroid, Papillary thyroid microcarcinoma, Central lymph node, Central lymph node dissection, Predictive factor, Scoring system

\section{Background}

Papillary thyroid microcarcinoma (PTMC) is defined as a papillary thyroid carcinoma measuring $1.0 \mathrm{~cm}$ or less in maximal diameter [1]. In the past, most patients with PTMC were diagnosed from specimens of thyroid removed for benign diseases, such as Graves' disease, follicular adenoma, and multinodular goiter. In recent years, due

\footnotetext{
*Correspondence: lasky1106@126.com

Ye-feng Cai and Qing-xuan Wang are co-first authors.

'Department of Oncology, the First Affiliated Hospital of Wenzhou Medical University, Wenzhou Medical University, No. 2 Fuxue Lane, Wenzhou 325000, China

Full list of author information is available at the end of the article
}

to the use of ultrasonography (US) and US-guided fineneedle aspiration cytology (FNAC), the number of patients with PTMC has been increasing. Although PTMC is usually associated with a favorable prognosis, regional lymph node metastasis (LNM), particularly in the central cervical compartment, is not uncommon [2]. The central lymph nodes are considered as the first echelon of lymphatic metastasis and are the level most frequently involved in papillary thyroid carcinoma (PTC) [3]. Recent studies demonstrated that the presence of nodal involvement was commonly associated with local recurrence and cancerspecific mortality $[4,5]$. Therefore, it is crucial to detect 
the metastatic lymph nodes preoperatively for PTMC, especially the central lymph nodes.

Nevertheless, the central lymph node metastasis (CLNM) is frequently diagnosed with microscopic LNM by pathology (metastatic deposits within a lymph node of less than $2 \mathrm{~mm}$ in maximal diameter) which is not easily detected preoperatively $[6,7]$. In clinical node-negative (cN0) PTMC, the presence of microscopic LNM is frequently detected in surgical specimens, and the prevalence of subclinical CLNM ranges from $30 \%$ to approximately $65 \%[2,8]$. Therefore, it is generally agreed that prophylactic central lymph node dissection ( $\mathrm{pCLND}$ ) should be performed to remove microscopic LNM because this treatment reduced local recurrence and cancer-specific mortality. However, pCLND remains a controversial subject. Zetoune et al. [9] showed that pCLND did not greatly reduce local recurrence in thyroid cancer. Carling et al. [10] demonstrated that the pCLND would lead to higher rates of hypoparathyroidism and recurrent laryngeal nerve injury. Moreover, a major argument against pCLND was a possibly increased risk of operative complications and medical cost which added the burden of patients. A prospective randomized controlled single-institution study demonstrated that cN0 patients with PTC treated with PCLND showed a similar outcome. Although one advantage pCLND could reduce necessity to repeat (131) I treatments, the disadvantage was a higher prevalence of permanent hypoparathyroidism. Actually, the different findings on this studies of pCLND could be explained with the variant percents of CLNM in different studies. Almost all clinicians agreed that central lymph node dissection (CLND) should be done with evidence of CLNM preoperatively. Hence, how to evaluate accurately the status of CLNM and choose the CLND as an optimum operation plan for PTMC are very important in thyroid operation.

Our study focused on investigating the relationship between CLNM and the clinicopathological and US characteristics to find the risk predictors for CLNM in PTMC. In addition, using the standardized regression coefficient, we developed a scoring system to identify the risk of CLNM and evaluate the predictive value of the scoring system.

\section{Methods}

\section{Patient}

We conducted a retrospective case-control study involving 498 patients who underwent total thyroidectomy or lobectomy with prophylactic central lymph node at the First Affiliated Hospital of Wenzhou Medical University from January 2009 to December 2012. All patients had no history of thyroid or neck surgery for nonthyroidial cancer, as well as of neck irradiation. The patients were divided into the case group (252 patients) and the control group (246 patients), according to the status of CLNM.
The surgical procedures were performed in the same operation team of the department of surgical oncology. The patients' clinical data were obtained from their electronic medical records. The research protocol used in this study was approved by the Ethics Committee of the First Affiliated Hospital of Wenzhou Medical University, and informed consent was obtained from each patient.

\section{Parameters analyzed}

Clinicopathologic and US characteristics were used to analyze risk factors of CLNM: a total of 16 selected factors in Table 1. All pathological diagnoses were made by an experienced pathologist. Primary tumor size based on US was measured by the standard of change in echo edge. When multiple PTMC were found in the specimen, the largest tumor or the most suspicious dominant nodule was analyzed. We used the age of 45 years as the cutoff because of its wide use as a clinical factor for prognosis. When multiple PTC were found in the specimen, the largest tumor or the most suspicious dominant nodule was analyzed. US calcification was defined as the hyperechoic spots with or without acoustic shadows or as the simple fine acoustic shadows in ultrasound [11]. ETE was defined as the tumor perimeter in contact with $>25 \%$ of the thyroid capsule in a malignant lesion or the loss of the capsule line according to the ultrasound [12]. Tumor location was classified in four areas (upper, middle, lower, and isthmus) based on preoperative ultrasonographic findings. LNM was obtained from the final pathologic reports. All the ultrasound features of the thyroid nodules were retrospectively reviewed by two experienced radiologists.

\section{Statistical analysis}

Data on normal distribution were expressed as mean \pm standard variation (SD) and were compared with $t$ test. Categorical variables were expressed as percentage and were compared with chi-square test or Fisher's exact test, as appropriate. Logistic regression analysis was also performed to estimate the odds ratios (OR) of certain parameters. Variables with $p<0.05$ in the univariate analysis were progressed to a multivariate analysis using forward stepwise selection. The standardized regression coefficient was used to quantify the discriminating power of each factor and to construct a 10-point score system. The best point for the score system was performed by receiver-operating characteristic (ROC) curves. All $p$ values were two sided, and a $p$ value of $<0.05$ was considered statistically significant. Statistical analysis was performed with SPSS software version 19.0 (SPSS, Chicago, IL, USA). 
Table 1 Baseline characteristics of papillary thyroid microcarcinoma patients stratified by the status of metastatic central lymph node

\begin{tabular}{|c|c|c|c|c|}
\hline \multirow[t]{2}{*}{ Characteristics } & \multirow[t]{2}{*}{ Total } & \multicolumn{2}{|c|}{ The status of metastatic central lymph node } & \multirow[t]{2}{*}{$p$ value } \\
\hline & & Positive & Negative & \\
\hline Total & 498 & 252 & 246 & \\
\hline Sex & & & & 0.130 \\
\hline Male $(n, \%)$ & $143(28.7 \%)$ & $80(31.7 \%)$ & $63(25.6 \%)$ & \\
\hline Female $(n, \%)$ & $355(71.3 \%)$ & $172(68.3 \%)$ & $183(74.4 \%)$ & \\
\hline Age at diagnosis (years) & & $45.8 \pm 9.8$ & $47.2 \pm 11.9$ & 0.150 \\
\hline$<45(n, \%)$ & $251(50.4 \%)$ & $136(54.0 \%)$ & $115(46.7 \%)$ & 0.107 \\
\hline$\geq 45(n, \%)$ & $247(49.6 \%)$ & $116(46.0 \%)$ & $131(53.3 \%)$ & \\
\hline Tumor size (mm) & & $6.8 \pm 2.1$ & $5.7 \pm 2.6$ & $<0.001$ \\
\hline$\leq 5(n, \%)$ & $165(33.1 \%)$ & $51(20.2 \%)$ & $114(46.3 \%)$ & $<0.001$ \\
\hline$>5(n, \%)$ & $333(66.9 \%)$ & $201(79.8 \%)$ & $132(53.7 \%)$ & \\
\hline Tumor location & & & & $<0.001$ \\
\hline Lower $(n, \%)$ & $141(28.3 \%)$ & $93(36.9 \%)$ & $48(19.5 \%)$ & \\
\hline Upper/middle/isthmus ( $n, \%)$ & $357(71.7 \%)$ & $159(63.1 \%)$ & $198(80.5 \%)$ & \\
\hline No. of nodules & & & & 0.416 \\
\hline Single $(n, \%)$ & $431(86.5 \%)$ & $215(85.3 \%)$ & $216(87.8 \%)$ & \\
\hline Multiple $(n, \%)$ & $67(13.5 \%)$ & $37(14.7 \%)$ & $30(12.2 \%)$ & \\
\hline Hashimoto's thyroiditis & & & & 0.348 \\
\hline Yes $(n, \%)$ & $87(17.5 \%)$ & $48(19.0 \%)$ & 39 (15.9\%) & \\
\hline No $(n, \%)$ & $411(82.5 \%)$ & $204(81.0 \%)$ & 207 (84.1 \%) & \\
\hline Extrathyroidal extension & & & & $<0.001$ \\
\hline Yes $(n, \%)$ & $249(50.0 \%)$ & $150(59.5 \%)$ & 99 (40.2\%) & \\
\hline No $(n, \%)$ & $249(50.0 \%)$ & $102(40.5 \%)$ & $147(59.8 \%)$ & \\
\hline Vascularization & & & & 0.29 \\
\hline Absence $(n, \%)$ & $435(87.3 \%)$ & $225(89.3 \%)$ & $210(85.4 \%)$ & \\
\hline External vascularization $(n, \%)$ & $31(6.2 \%)$ & $15(6.0 \%)$ & $16(6.5 \%)$ & \\
\hline Internal vascularization $(n, \%)$ & $32(6.4 \%)$ & $12(4.8 \%)$ & $20(8.1 \%)$ & \\
\hline Intact capsule & & & & 0.548 \\
\hline Yes $(n, \%)$ & $297(59.6 \%)$ & $147(58.3 \%)$ & $150(61.0 \%)$ & \\
\hline No $(n, \%)$ & $201(40.4 \%)$ & $105(41.7 \%)$ & 96 (39.0 \%) & \\
\hline Margin & & & & $<0.001$ \\
\hline Well-defined $(n, \%)$ & $246(89.8 \%)$ & 99 (39.3 \%) & $147(59.8 \%)$ & \\
\hline No well-defined $(n, \%)$ & $252(50.6 \%)$ & $153(60.7 \%)$ & 99 (40.2\%) & \\
\hline Shape & & & & 0.280 \\
\hline Regular ( $n, \%)$ & $372(74.7 \%)$ & $183(72.6 \%)$ & $189(76.8 \%)$ & \\
\hline Irregular $(n, \%)$ & $126(25.3 \%)$ & $69(27.4 \%)$ & $57(23.2 \%)$ & \\
\hline Composition & & & & 0.188 \\
\hline Solid $(n, \%)$ & $435(87.3 \%)$ & $225(89.3 \%)$ & $210(85.4 \%)$ & \\
\hline Cystic or mixed $(n, \%)$ & $63(12.7 \%)$ & $27(10.7 \%)$ & $36(14.6 \%)$ & \\
\hline Calcification & & & & $<0.001$ \\
\hline Absence $(n, \%)$ & $237(47.6 \%)$ & $102(40.5 \%)$ & 135 (54.9 \%) & \\
\hline Microcalcification $(n, \%)$ & $207(41.6 \%)$ & $129(51.2 \%)$ & 78 (31.7 \%) & \\
\hline
\end{tabular}


Table 1 Baseline characteristics of papillary thyroid microcarcinoma patients stratified by the status of metastatic central lymph node (Continued)

\begin{tabular}{|c|c|c|c|c|}
\hline Other calcification $(n, \%)$ & $54(10.8 \%)$ & $21(8.3 \%)$ & $33(13.4 \%)$ & \\
\hline Taller than wide & & & & 0.916 \\
\hline Yes $(n, \%)$ & $432(86.7 \%)$ & $219(86.9 \%)$ & $213(86.6 \%)$ & \\
\hline No $(n, \%)$ & $66(13.3 \%)$ & $33(13.1 \%)$ & $33(13.4 \%)$ & \\
\hline Display of enlarged lymph nodes & & & & $<0.001$ \\
\hline Yes $(n, \%)$ & $267(53.6 \%)$ & $165(65.5 \%)$ & $102(41.5 \%)$ & \\
\hline No $(n, \%)$ & $231(46.4 \%)$ & $87(34.5 \%)$ & $144(58.5 \%)$ & \\
\hline Contact of $>25 \%$ with the adjacent capsule & & & & $<0.001$ \\
\hline Yes $(n, \%)$ & $153(30.7 \%)$ & 105 (41.7 \%) & $48(19.5 \%)$ & \\
\hline No $(n, \%)$ & $345(69.3 \%)$ & $147(58.3 \%)$ & $198(80.5 \%)$ & \\
\hline
\end{tabular}

\section{Results}

\section{Univariate analysis}

According to the status of metastatic central lymph node, patients were divided into two groups (the case group: CLNM positive; the control group: CLNM negative). Differences in clinicopathological and US features between two groups were compared in Table 1. Seven factors were significantly associated with CLNM: display of enlarged lymph node $(p<0.001)$, contact of $>25 \%$ with the adjacent capsule $(p<0.001)$, no well-defined margin $(p<0.001)$, tumor size $(p<0.001)$, tumor location $(p<0.001)$, extrathyroidal extension $(p<0.001)$, and calcification $(p<0.001)$.

\section{Multivariate analysis}

In the multivariate logistic regression, tumor size $>5 \mathrm{~mm}$ (OR 2.986; $p<0.001 ; 95 \%$ confidence interval (CI) 1.8614.790; standardized coefficient regression (Coef) 1.094), the lower polo (OR 3.568; $p<0.001$; $95 \%$ CI 2.211-5.758; Coef 1.272), extrathyroidal extension (OR 2.631; $p<0.001$; 95 \% CI 1.720-4.023; Coef 0.967), no well-defined margin (OR 2.459; $p<0.001 ; 95 \%$ CI 1.611-3.754; Coef 0.9), display of metastatic lymph node (OR 3.01; $p<0.001$; $95 \%$ CI 1.954-4.634; Coef 1.102), and contact of $>25 \%$ with the adjacent capsule (OR 2.471; $p<0.001 ; 95 \%$ CI 1.556-3.922; Coef 0.904) had highly independent association with central LNM. The relevant information is shown in Table 2.

\section{A scoring system}

As shown in Table 2, the standardized regression coefficient is used to quantify the discriminating power (Coef value) of six predictors and construct a 10-point scoring system based on their relative predictive weight. The sum of the points was evaluated to distinguish between patients with and without CLNM. The mean index points were $5.71 \pm 1.72$ in the CLNM-positive group and $3.55 \pm 1.95$ in the CLNM-negative group, which showed statistically significant difference $(p<0.001)$. Using ROC curve, the area under the ROC curve was 0.791 (Fig. 1). Then, the cutoff value of 5 was found to be the best points. The sensitivity and specificity for this scoring system were 64.7 and $80.5 \%$, respectively, and the positive predictive value and negative predictive value were 77.2 and $69.0 \%$, respectively. When comparing to other single independent predictors, the scoring system had more satisfied predictive value (Table 3 ).

\section{Discussion}

In our study, we listed a total of 16 potential predictors according to the clinicopathological and US features. Using the univariate analysis, we found that seven predicting factors were significantly associated with the status of

Table 2 Multivariate analysis (logistic regression) and 10-point scoring system

\begin{tabular}{|c|c|c|c|c|c|}
\hline Characteristic & OR & $95 \% \mathrm{Cl}$ & $p$ value & Coef & Score \\
\hline Size & 2.986 & $1.861-4.790$ & $<0.001$ & 1.094 & 1.75 \\
\hline Tumor location & 3.568 & $2.211-5.758$ & $<0.001$ & 1.272 & 2.00 \\
\hline Extrathyroidal extension & 2.631 & $1.720-4.023$ & $<0.001$ & 0.967 & 1.55 \\
\hline Margin & 2.459 & $1.611-3.754$ & $<0.001$ & 0.900 & 1.45 \\
\hline Display of enlarged lymph nodes & 3.010 & $1.954-4.634$ & $<0.001$ & 1.102 & 1.75 \\
\hline Contact of $>25 \%$ with the adjacent capsule & 2.471 & $1.556-3.922$ & $<0.001$ & 0.904 & 1.50 \\
\hline
\end{tabular}

Coef standardized coefficient regression, OR odds ratio, $95 \% \mathrm{Cl} 95 \%$ confidence interval 


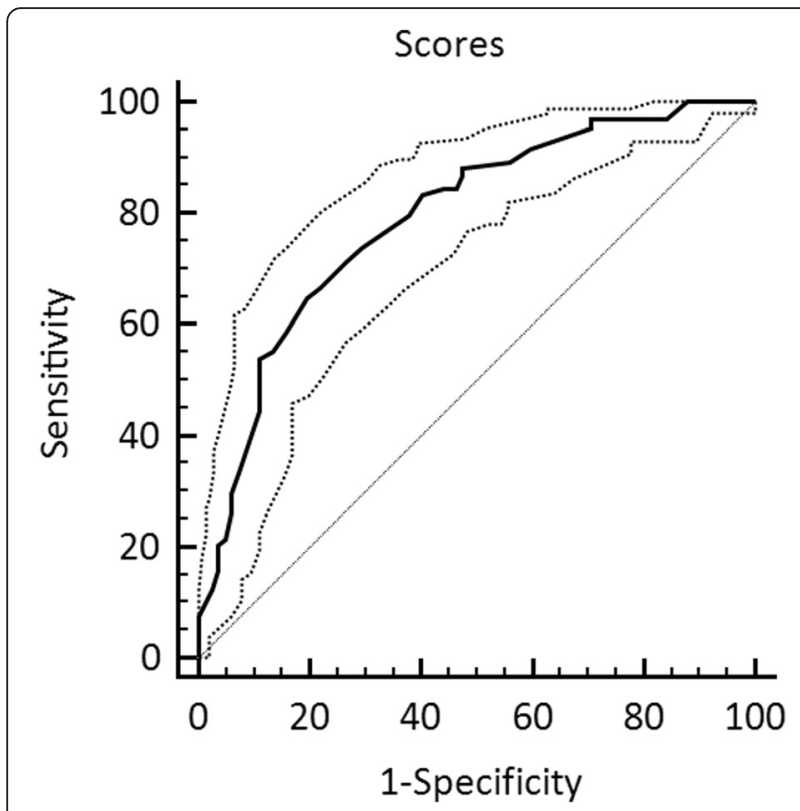

Fig 1 Receiver-operating characteristic (ROC) curve for the scoring system. The area under the ROC curve was 0.791. According to the ROC curve, a cutoff value of 5 points was found to be best for distinguishing between patients without and with central LNM. The sensitivity and specificity were 64.7 and $80.5 \%$, respectively, and the positive predictive value and negative predictive value were 77.2 and $69.0 \%$, respectively. The dotted line represents the $95 \% \mathrm{Cl}$

central LNM. In addition, six independent predictors of tumor size $(>5 \mathrm{~mm})$, tumor location (the lower polo), extrathyroidal extension, margin (no well-defined), display of enlarged lymph node, and contact of $>25 \%$ with the adjacent capsule for CLNM were confirmed by multivariate logistic regression analysis.

The incidence of CLNM in PTMC patients has been reported ranging from 24.1 to $64.1 \%$ [2, 8]. In this study, we found that the CLNM rate was $50.6 \%$, which was in accordance with the incidence rate.

Wada et al. [2] had reported that the location of PTMC within the thyroid was related to the prevalence of CLNM. $\mathrm{Xu}$ et al. [13] found that a tumor located in the lower third of the thyroid lobe was a high-risk factor of central lymph node metastasis. Zhang et al. [14] showed that the patients with a tumor located in the upper pole of the thyroid lobe conferred a lower risk for CLNM and a higher risk for lateral cervical metastasis. Similarly, we discovered that tumor location was an independent predictor for CLNM. The tumor located in the lower polo of the thyroid has a higher risk for CLNM, while the tumor located in the upper third may be a lower risk for CLNM.

Moon et al. [15] certified that PTMC with minimal ETE more frequently had CLNM than without. He et al. [16] found that extrathyroidal extension was associated with subclinical CLNM. Varshney et al. [17] declared the ETE was associated with the CLNM of PTMC. In agreement with previous results, we found that thyroid nodules with ETE were an independent predictive factor for CLNM.

Generally, no well-defined margin of the thyroid nodule has the strongest risk of malignance. Ito et al. [18] suggested that no well-defined margin was an important US feature of biologically aggressive PTMC. Also, we found that the margin was a highly independent predictor for CLNM in PTMC.

Traditionally, patients who have PTMC presenting with palpable lymphadenopathy, by preoperative US, should have cervical node dissection [2]. PTMC with ultrasonographical display of metastatic lymph nodes, especially enlarged lymph nodes, was more likely to recur in the regional lymph nodes [19]. According to the univariate and multivariate analyses, we found that display of enlarged lymph nodes was an independent predictor for CLNM.

Our study found contact of $>25 \%$ with the adjacent capsule was an independent predicting factor for CLNM. This US feature is a very important and visualized in ultrasonic examination. Many literatures suggest that the presence of contact with the adjacent capsule can provide a useful predictive information about ETE [20, 21]. According to the above analysis, about the relation between ETE and CLNM, there was an indirect relationship between contact of $>25 \%$ with the adjacent capsule and CLNM.

Many literatures confirmed that tumor size was associated with CLNM. [2, 22]. Many literatures confirmed

Table 3 The predictive value for the scoring system and independent predictors

\begin{tabular}{|c|c|c|c|c|}
\hline Independent predictors & Sensitivity (\%) & Specificity (\%) & +PV (\%) & $-P V(\%)$ \\
\hline Size & 79.8 & 46.3 & 60.4 & 69.1 \\
\hline Tumor location & 36.9 & 80.5 & 66.0 & 55.5 \\
\hline Extrathyroidal extension & 59.5 & 62.6 & 63.0 & 59.0 \\
\hline Margin & 60.7 & 59.8 & 60.7 & 59.8 \\
\hline Display of enlarged lymph nodes & 65.5 & 58.5 & 61.8 & 62.3 \\
\hline Contact of $>25 \%$ with the adjacent capsule & 41.7 & 80.5 & 68.6 & 57.4 \\
\hline The scoring system & 64.7 & 80.5 & 77.3 & 69.0 \\
\hline
\end{tabular}

$+P V$ positive predictive value, $-P V$ negative predictive value 
that tumor size has significant relation with CLNM in PTMC using the cutoff value of $5 \mathrm{~mm}[13,23,24]$. Therefore, in the present study, the tumor size of $5 \mathrm{~mm}$ was regarded as the cutoff value and was proved as an independent predictor for CLNM.

In this study, calcification was not an independent factor for central LNM, although it was statistically significant on the univariate analysis, which was similar to two previous reports [20,25]. Age is known to be an important prognostic factor for patients with PTC. However, its predictive value in PTMC was uncertain $[2,23]$. In our study, the gender has no association with CLNM. Similarly, Liu et al. [23] demonstrate that there was no relation observed between CLNM and gender in a meta-analysis. The association between multifocality and CLNM was still debatable [22, 23, 26]. In our study, no relevance was found between them. The main reason may be explained that the incidence rate of multifocality in the present study is very lower than other studies.

The role of pCLND was still a debate. In our opinion, those previous studies which analyzed the clinical results of pCLND had a deficiency. When assessing complication rates after thyroidectomy, most data are from highvolume endocrine surgery centers of excellence where the surgeons have more operation experience and lower complications rates [10]. In other words, the surgeons' operation experience on CLND and hospital level had important influence on survival, postoperative region recurrence, and complications in PTMC. Hence, the issue of pCLND is likely to remain so.

If the CLNM could be diagnosed definitively preoperatively, the CLND can be easily established. However, in many patients, CLNM may not show any abnormal finding in preoperative imaging examinations due to their limitation. To improve the diagnostic accuracy, we used a combination of clinicopathologic and US characteristics to find six independent predictors for CLNM and developed a scoring system for predicting CLNM in PTMC. Using ROC curves, we identified the cutoff value of 5 points as the best score for detection of CLNM with a sensitivity of $64.7 \%$, a specificity of $80.5 \%$, positive predictive value of $77.2 \%$, and negative predictive value of $69.0 \%$. The scoring system has obvious advantages in predicting CLNM in PTMC relative to other single factor. Moreover, the predictors in this scoring system mainly come from US characteristics, which is convenient and feasible for predicting CLNM.

At present, there are many PTC-specific mutations, such as BRAF V600E and TERT promoter, which can predict the risk of CLNM in PTMC [27-29]. However, in Asia, most countries are the developing countries and their medical levels are lower than Europe and the USA. Patients often cannot afford to take more medical examination like molecular test. Therefore, in many Asian developing countries, the scoring system we raised has the advantages of simplicity and high sensitivity and specificity (compared with ultrasound) and will be helpful for surgeons to decide the essential surgical strategy for patients with PTMC, in particular the subclinical CLNM. In addition, we think that the use of this scoring system will reduce the surgical complications and develop the individual therapy for PTMC.

However, there are several potential limitations in this study. Firstly, the PTMC has very favorable prognosis and the follow-up time would be at least 10 years. Our study did not analyze data from the long-term follow-up period, such as disease recurrence and disease-free survival; thus, we cannot directly conclude that the scoring system can predict the prognosis. Secondly, this study was conducted in a cross-sectional design, rather than longitudinal observations. Thirdly, single-center verification is not accurate enough. The scoring system needs multicenter validation to check its predictive value. Further investigation with a longer duration is needed.

\section{Conclusions}

In conclusion, we first established a scoring system for predicting CLNM preoperatively in PTMC. The scoring system of clinicopathological and US characteristics for the prediction of CLNM can be another alternative diagnostic approach when the results of preoperative diagnostic evaluation for CLNM are not adequate or obvious. The points $\leq 5$ could be considered as a low risk for CLNM, and the points $>5$ could be considered as a high risk for CLNM. More aggressive diagnostic approaches and prophylactic CLND are needed for the points $>5$. Therefore, the scoring system will be helpful for surgeons to decide the most suitable surgical strategy for patients with PTMC, especially the subclinical CLNM.

\section{Abbreviations \\ CLND: central lymph node dissection; CLNM: central lymph node metastasis; FNAC: fine-needle aspiration cytology; PCLND: prophylactic central lymph node} dissection; PTMC: papillary thyroid microcarcinoma; US: ultrasonography.

\section{Competing interests}

The authors declare that they have no competing interests.

\begin{abstract}
Authors' contributions
$X-H Z, Y-F C, Q L$ and $C-J N$ designed the study. C-JN, Q-XW, H-MZ, and X-JZ collected the data. Y-FC, E-DC, and QL did the statistical analyses. Y-FC, C-JN, S-YD, X-HZ and QL reviewed the results, interpreted the data, and wrote the manuscript. All authors saw and approved the final version of the paper.
\end{abstract}

\section{Acknowledgements}

This work was supported by a grant from the National High Technology Research and Development Program of 863 project of China (NO. 2012AA02A210), Scientific Research Incubator Project of the First Affiliated Hospital of Wenzhou Medical University (NO. FHY2014018), and the Wenzhou City Science and Technology Bureau (NO. Y20140136). 


\section{Author details}

'Department of Oncology, the First Affiliated Hospital of Wenzhou Medical University, Wenzhou Medical University, No. 2 Fuxue Lane, Wenzhou 325000, China. ${ }^{2}$ Department of Anesthesiology, the First Affiliated Hospital of Wenzhou Medical University, Wenzhou 325000, China. ${ }^{3}$ Department of Diagnostic Ultrasound, the First Affiliated Hospital of Wenzhou Medical University, Wenzhou 325000, China.

Received: 21 August 2015 Accepted: 17 February 2016

Published online: 24 February 2016

\section{References}

1. Sobin LH. Histological typing of thyroid tumours. Histopathology. 1990;16(5):513.

2. Wada N, Duh QY, Sugino K, Iwasaki H, Kameyama K, Mimura T, et al. Lymph node metastasis from 259 papillary thyroid microcarcinomas: frequency, pattern of occurrence and recurrence, and optimal strategy for neck dissection. Ann Surg. 2003;237(3):399-407. doi:10.1097/01.SLA.0000055273.58908.19.

3. Noguchi S, Noguchi A, Murakami N. Papillary carcinoma of the thyroid. I. Developing pattern of metastasis. Cancer. 1970;26(5):1053-60.

4. Ito Y, Tomoda C, Uruno T, Takamura Y, Miya A, Kobayashi K, et al. Ultrasonographically and anatomopathologically detectable node metastases in the lateral compartment as indicators of worse relapse-free survival in patients with papillary thyroid carcinoma. World J Surg. 2005; 29(7):917-20. doi:10.1007/s00268-005-7789-x

5. Cho SY, Lee TH, Ku YH, Kim HI, Lee GH, Kim MJ. Central lymph node metastasis in papillary thyroid microcarcinoma can be stratified according to the number, the size of metastatic foci, and the presence of desmoplasia. Surgery. 2015;157(1):111-8. doi:10.1016/j.surg.2014.05.023.

6. Mulla M, Schulte KM. Central cervical lymph node metastases in papillary thyroid cancer: a systematic review of imaging-guided and prophylactic removal of the central compartment. Clin Endocrinol. 2012;76(1):131-6. doi:10.1111/j.1365-2265.2011.04162.x.

7. Stulak JM, Grant CS, Farley DR, Thompson GB, van Heerden JA, Hay ID, et al. Value of preoperative ultrasonography in the surgical management of initial and reoperative papillary thyroid cancer. Arch Surg. 2006;141(5):489-94. doi:10.1001/archsurg.141.5.489. discussion 94-6.

8. Roh JL, Kim JM, Park Cl. Central cervical nodal metastasis from papillary thyroid microcarcinoma: pattern and factors predictive of nodal metastasis. Ann Surg Oncol. 2008:15(9):2482-6. doi:10.1245/s10434-008-0044-6.

9. Zetoune T, Keutgen X, Buitrago D, Aldailami H, Shao H, Mazumdar M, et al. Prophylactic central neck dissection and local recurrence in papillary thyroid cancer: a meta-analysis. Ann Surg Oncol. 2010;17(12):3287-93. doi:10.1245/s10434-010-1137-6.

10. Carling T, Long 3rd WD, Udelsman R. Controversy surrounding the role for routine central lymph node dissection for differentiated thyroid cancer. Curr Opin Oncol. 2010;22(1):30-4. doi:10.1097/CCO.0b013e328333ac97.

11. Solbiati L, Cioffi V, Ballarati E. Ultrasonography of the neck. Radiol Clin N Am. 1992;30(5):941-54.

12. Moon HJ, Kim EK, Yoon JH, Kwak JY. Differences in the diagnostic performances of staging US for thyroid malignancy according to experience. Ultrasound Med Biol. 2012;38(4):568-73. doi:10.1016/j.ultrasmedbio.2012.01.002.

13. Xu D, Lv X, Wang S, Dai W. Risk factors for predicting central lymph node metastasis in papillary thyroid microcarcinoma. Int J Clin Exp Pathol. 2014;7(9):6199-205

14. Zhang L, Wei WJ, Ji QH, Zhu YX, Wang ZY, Wang Y, et al. Risk factors for neck nodal metastasis in papillary thyroid microcarcinoma: a study of 1066 patients. J Clin Endocrinol Metab. 2012;97(4):1250-7. doi:10.1210/jc.2011-1546.

15. Moon HJ, Kim EK, Chung WY, Yoon JH, Kwak JY. Minimal extrathyroidal extension in patients with papillary thyroid microcarcinoma: is it a real prognostic factor? Ann Surg Oncol. 2011;18(7):1916-23. doi:10.1245/s10434-011-1556-Z.

16. He Q, Zhuang D, Zheng L, Fan Z, Zhou P, Lv Z, et al. The surgical management of papillary thyroid microcarcinoma: a 162-month single-center experience of 273 cases. Am Surg. 2012;78(11):1215-8.

17. Varshney R, Pakdaman MN, Sands N, Hier MP, Rochon L, Black MJ, et al. Lymph node metastasis in thyroid papillary microcarcinoma: a study of 170 patients. J Laryngol Otol. 2014;128(10):922-5. doi:10.1017/S0022215114001704.

18. Ito Y, Kobayashi K, Tomoda C, Uruno T, Takamura Y, Miya A, et al. Ill-defined edge on ultrasonographic examination can be a marker of aggressive characteristic of papillary thyroid microcarcinoma. World J Surg. 2005; 29(8):1007-11. doi:10.1007/s00268-005-7834-9. discussion 11-2.
19. Ito $Y$, Miyauchi A. A therapeutic strategy for incidentally detected papillary microcarcinoma of the thyroid. Nat Clin Pract Endocrinol Metab. 2007;3(3):240-8. doi:10.1038/ncpendmet0428.

20. Kim SS, Lee BJ, Lee JC, Kim SJ, Lee SH, Jeon YK, et al. Preoperative ultrasonographic tumor characteristics as a predictive factor of tumor stage in papillary thyroid carcinoma. Head Neck. 2011;33(12):1719-26. doi:10.1002/hed.21658

21. Kwak JY, Kim EK, Youk JH, Kim MJ, Son EJ, Choi SH, et al. Extrathyroid extension of well-differentiated papillary thyroid microcarcinoma on US. Thyroid. 2008;18(6):609-14. doi:10.1089/thy.2007.0345.

22. Lee SH, Lee SS, Jin SM, Kim JH, Rho YS. Predictive factors for central compartment lymph node metastasis in thyroid papillary microcarcinoma. Laryngoscope. 2008;118(4):659-62. doi:10.1097/MLG.0b013e318161f9d1.

23. Liu Z, Wang L, Yi P, Wang CY, Huang T. Risk factors for central lymph node metastasis of patients with papillary thyroid microcarcinoma: a meta-analysis. Int J Clin Exp Pathol. 2014;7(3):932-7.

24. Kim KE, Kim EK, Yoon JH, Han KH, Moon HJ, Kwak JY. Preoperative prediction of central lymph node metastasis in thyroid papillary microcarcinoma using clinicopathologic and sonographic features. World J Surg. 2013;37(2):385-91. doi:10.1007/s00268-012-1826-3.

25. Yoo YH, Kim JA, Son EJ, Youk JH, Kwak JY, Kim EK, et al. Sonographic findings predictive of central lymph node metastasis in patients with papillary thyroid carcinoma: influence of associated chronic lymphocytic thyroiditis on the diagnostic performance of sonography. J Ultrasound Med. 2013;32(12):2145-51. doi:10.7863/ultra.32.12.2145.

26. Lim YC, Choi EC, Yoon YH, Kim EH, Koo BS. Central lymph node metastases in unilateral papillary thyroid microcarcinoma. Br J Surg. 2009;96(3):253-7. doi:10.1002/bjs.6484.

27. Lin KL, Wang OC, Zhang XH, Dai XX, Hu XQ, Qu JM. The BRAF mutation is predictive of aggressive clinicopathological characteristics in papillary thyroid microcarcinoma. Ann Surg Oncol. 2010;17(12):3294-300. doi:10.1245/s10434-010-1129-6.

28. Yang $Y$, Chen $C$, Chen Z, Jiang J, Chen $Y$, Jin $L$, et al. Prediction of central compartment lymph node metastasis in papillary thyroid microcarcinoma. Clin Endocrinol. 2014;81(2):282-8. doi:10.1111/cen.12417.

29. De-Tao $Y$, Kun $Y$, Run-Qing $L$, Xianghua $L$, Jianhui $X$, Mengyuan $L$ et al. Clinicopathological significance of TERT promoter mutation in papillary thyroid carcinomas: a systematic review and meta-analysis. Clin Endocrinol. 2016. doi:10.1111/cen.13017

\section{Submit your next manuscript to BioMed Central and we will help you at every step:}

- We accept pre-submission inquiries

- Our selector tool helps you to find the most relevant journal

- We provide round the clock customer support

- Convenient online submission

- Thorough peer review

- Inclusion in PubMed and all major indexing services

- Maximum visibility for your research

Submit your manuscript at www.biomedcentral.com/submit
) Biomed Central 